\title{
Distinctive distribution of lymphocytes in unruptured and previously untreated brain arteriovenous malformation
}

\author{
Yi Guo ${ }^{1,6}$, Tarik Tihan², Helen Kim¹, Christopher Hess ${ }^{3}$, Michael T. Lawton ${ }^{4}$, William L. Young ${ }^{1,4,5}$, \\ Yuan-Li Zhao ${ }^{7}$, Hua Su ${ }^{1}$ \\ ${ }^{1}$ Department of Anaesthesia and Perioperative Care, Center for Cerebrovascular Research, San Francisco, CA 94110 , USA. \\ ${ }^{2}$ Department of Pathology, University of California, San Francisco, San Francisco, CA 94110, USA. \\ ${ }^{3}$ Department of Radiology and Biomedical Imaging, University of California, San Francisco, San Francisco, CA 941 10 , USA. \\ ${ }^{4}$ Department of Neurological Surgery, University of California, San Francisco, San Francisco, CA 94110, USA. \\ ${ }^{5}$ Department of Neurology, University of California, San Francisco, San Francisco, CA 94110, USA. \\ ${ }^{6}$ Department of Neurosurgery, Affiliated Hospital of Hebei University, Baoding 071000, Hebei, China. \\ ${ }^{7}$ Department of Neurosurgery, Beijing Tiantan Hospital, Capital Medical University, Beijing 410011, China.
}

\section{A B S T R A C T}

Aim: To test the hypothesis that lymphocyte infiltration in brain arteriovenous malformation (bAVM) is not associated with iron deposition (indicator of micro-hemorrhage). Methods: Sections of unruptured, previously untreated bAVM specimens $(n=19)$ were stained immunohistochemically for T-lymphocytes (CD3 $\left.{ }^{+}\right)$, B-lymphocytes $\left(C D 20^{+}\right)$, plasma cells $\left(C D 138^{+}\right)$and macrophages $\left(C D 68^{+}\right)$. Iron deposition was assessed by hematoxylin and eosin and Prussian blue stains. Superficial temporal arteries (STA) were used as control. Results: Both T-lymphocytes and macrophages were present in unruptured, previously untreated bAVM specimens, whereas few B cells and plasma cells were detected. Iron deposition was detected in 8 specimens (42\%; 95\% confidence intervals $=20-67 \%$ ). The samples with iron deposition tended to have more macrophages than those without $\left(666 \pm 313 \mathrm{vs} .478 \pm 174 \mathrm{cells} / \mathrm{mm}^{2} ; P=0.11\right)$. T-cells were clustered on the luminal side of the endothelial surface, on the vessel-wall, and in the perivascular regions. There was no correlation between T-lymphocyte load and iron deposition $(P=0.88)$. No macrophages and lymphocytes were detected in STA controls. Conclusion: T-lymphocytes were present in bAVM specimens. Unlike macrophages, the load and location of T-lymphocytes were not associated with iron deposition, suggesting the possibility of an independent cell-mediated immunological mechanism in bAVM pathogenesis.

Key words: B-lymphocyte, human brain arteriovenous malformation, inflammatory cells, micro-hemorrhage, T-lymphocyte

\section{INTRODUCTION}

Human brain arteriovenous malformations (bAVMs) are tangles of abnormal vessels between arteries and veins and lack of capillary bed. Brain AVM is the most common cause of hemorrhagic stroke in young adults and children. ${ }^{[1-3]}$ Commonly assumed to be congenital, postnatal formation may be more prevalent than previously thought, ${ }^{[4-6]}$ and the etiology of bAVMs still remains unclear. Genetic factors, ${ }^{[7,8]}$ aberrant vasculogenesis, ${ }^{\left[{ }^{-11]}\right.}$ and inflammation may all play roles

\begin{tabular}{|l|l|}
\hline \multicolumn{2}{|c|}{ Access this article online } \\
\hline Quick Response Code: & \multirow{2}{*}{ Website: } \\
\hline & www.nnjournal.net \\
\cline { 2 - 3 } & \\
\hline
\end{tabular}

in the pathogenesis of bAVMs; ${ }^{[12]}$ a confluence of these factors has been proposed in a "response-to-injury" paradigm. ${ }^{[5]}$

Evidence indicating the involvement of inflammation in bAVM pathogenesis includes neutrophil and macrophage infiltration, and increased expression of various inflammatory signals, such as matrix metalloproteinase-9, interleukin-6, myeloperoxidase and adhesion molecules. ${ }^{[13-18]}$ About half of bAVMs cases present with an intracranial hemorrhage (ICH), which itself can induce inflammation. However, even in unruptured and untreated AVMs, substantial infiltration of inflammatory cells has been detected in the vascular wall and intervening stroma. ${ }^{[13]}$ Magnetic resonance imaging has detected hemosiderin deposition in unruptured bAVMs, ${ }^{[19,20]}$ consistent with episodes of clinically silent intralesional micro-hemorrhage. 
We recently described a strong association between imaging evidence of old silent hemorrhage and the risk of clinically symptomatic ICH. ${ }^{[21]}$ Further, histological examination demonstrated that the degree of hemosiderin deposition is positively correlated with the number of macrophages in the lesion. ${ }^{[21]}$ It is not clear, however, whether the macrophage response is specific or whether other inflammatory cells are also correlated with hemosiderin deposition and macrophage. Our previous studies demonstrated that both macrophage and neutrophil may play roles in bAVM pathogenesis. ${ }^{[13-15]}$ Shi et al. described evidence of adaptive immunological responses in cavernous malformation. ${ }^{[22]}$ Although bAVM tissue was used as control in Shi's study and while no oligoclonal response was observed, bAVM had a higher polyclonal response compared to normal brain tissue, suggesting that lymphocytes may also play a role in bAVM.

In this study, we analyzed lymphocytes in addition to macrophages, and tested the hypothesis that, unlike the innate immune cells (macrophages), adaptive immune cell (lymphocytes)-infiltration is not associated with micro-hemorrhage and iron deposition.

\section{METHODS}

All studies involving patients were approved by the Institutional Review Board of the University of California, San Francisco (UCSF), and patients gave informed consent.

\section{Patients}

Patients with AVMs evaluated at UCSF have been entered into an ongoing prospective registry since 2000. ${ }^{[23]}$ We identified 24 unruptured brain AVMs from patients who did not undergo preoperative embolization or radiosurgery with frozen tissue available in our database; 19 samples were located and used in this study [Table 1]. Three superficial temporal arteries (STA), obtained from autopsies of patients who died from nonbrain-related diseases, were used as control.

\section{Histology}

Prussian blue staining was performed using Accustain Iron Stain kit (Sigma-Aldrich, St. Louis, MO) according to the manufacturer's protocol.

For immunohistochemistry, adjacent sections were used to stain different surface markers. CD68, CD3, CD20 and CD138-specific antibodies were purchased from Abcam (Abcam, Cambridge, MA). Brain AVM specimens were embedded in optimum cutting temperature, sectioned into $8 \mu \mathrm{m}$ sections, and fixed with $4 \%$ paraformaldehyde. Endogenous peroxidase

\begin{tabular}{lclcl}
\hline Table I: Patient and lesion characteristics & \\
Patients & $\begin{array}{c}\text { Age } \\
\text { (years) }\end{array}$ & Sex & $\begin{array}{c}\text { Size } \\
\text { (cm) }\end{array}$ & $\begin{array}{l}\text { Presentation/ } \\
\text { clinical details }\end{array}$ \\
\hline 1 & 38 & Female & 1 & \\
2 & 24 & Female & 3 & \\
3 & 37 & Male & 3 & Incidental \\
4 & 44 & Male & 1 & Seizure \\
5 & 54 & Female & 2 & \\
6 & 49 & Female & 1 & Seizure \\
7 & 63 & Male & 1 & \\
8 & 54 & Female & 3 & Seizure \\
9 & 47 & Female & 2 & \\
10 & 39 & Female & 4 & \\
11 & 53 & Female & 2 & Focal deficit \\
12 & 20 & Male & 3 & Incidental \\
13 & 53 & Male & 4 & Headache \\
14 & 30 & Male & 3 & Incidental \\
15 & 49 & Female & 1 & Incidental \\
16 & 41 & Male & 3 & \\
17 & 30 & Male & 4 & \\
18 & 30 & Female & 3 & Seizure \\
19 & 45 & Female & 2 & \\
\hline
\end{tabular}

activity was quenched by incubating slides in $0.3 \%$ $\mathrm{H}_{2} \mathrm{O}_{2}$ in phosphate-buffered saline (PBS) for $15 \mathrm{~min}$. After blocking with $10 \%$ normal donkey serum, sections were incubated at $4{ }^{\circ} \mathrm{C}$ overnight with primary antibodies diluted in PBS with 1\% BSA in the following concentrations: mouse monoclonal antihuman CD68, 1:1000; rabbit monoclonal antihuman CD3, 1:400; rabbit monoclonal antihuman CD20, 1:200; mouse monoclonal antihuman CD138, 1:800. After washing in PBS, the sections were incubated with horseradish peroxidase labeled antimouse or antirabbit IgG (vector labs) for $1 \mathrm{~h}$ at room temperature. The positive staining was visualized using 3, 3-diaminobenzidene. Negative controls were performed by omitting the primary antibodies during immunostaining.

The criteria for identifying hemosiderin were birefringent or brownish particles seen in the vascular wall or interstitial tissue between vessels, and were confirmed by Prussian blue staining on adjacent sections. $\mathrm{CD}^{2} 8^{+}, \mathrm{CD}^{+}, \mathrm{CD} 20^{+}, \mathrm{CD} 138^{+}$cells were quantified by counting the positively stained cells using stereological microscopy (Olympus, Japan).

\section{Statistical analysis}

All data are expressed as mean \pm standard deviation the differences of means were analyzed using unpaired Student's $t$-test. Exact binomial 95\% confidence intervals (CIs) for proportions are reported. $P<0.05$ was considered as statistically significant.

\section{RESULTS}

Hemosiderin deposition was present in unruptured brain arteriovenous malformations

Consistent with our published data, ${ }^{[21]}$ hemosiderin deposition was found in 8 out of 19 specimens 
(42\%; 95\% CI: 20-67\%) [Figure 1]. Hemosiderin positive cells were scattered mainly around the abnormal vessels [Figure 1a]. Prussian blue positive staining was detected in the areas that had hemosiderin deposition [Figure 1d], suggesting the presence of previous micro-hemorrhage.

T-lymphocytes and macrophages were detected in unruptured brain arteriovenous malformations

To analyze whether the lymphocytes were present in unruptured bAVM and whether their location was associated with macrophages and iron deposition, we analyzed T- and B-lymphocytes, plasma cells and macrophages. We found that T-lymphocyte was the predominant type of lymphocytes present in unruptured bAVM. Whereas the macrophages were scattered mostly in the vessel walls and intervening stromal regions [Figure 2], T-lymphocytes were clustered on the luminal side of the endothelial surface, in the vascular wall, and in the tissue between abnormal vessels [Figure 3]. Few B-lymphocytes were detected; they were mostly present in samples that had a large number of T-lymphocytes, and were co-localized with the T-lymphocytes [Figure 2]. In addition, a few plasma cells were identified in 5 samples, of which 4 had hemosiderin deposition (data not shown). No lymphocytes and macrophages were detected in STA [Figure 2].

Compared to the specimens that had no hemosiderin deposition, hemosiderin-positive specimens tended to have more macrophages (478 \pm 174 vs. $666 \pm 313$ cells $\left./ \mathrm{mm}^{2} ; P=0.11\right)$. The T-cell numbers were similar in hemosiderin-positive and hemosiderin-negative samples (147 \pm 108 vs. $157 \pm 139$ cells $/ \mathrm{mm}^{2} ; P=0.88$ ) [Figure 4].

\section{DISCUSSION}

We found in this study that T-cells are the predominant lymphocytes in unruptured bAVMs. Few B-lymphocytes and plasma cells were detected. Unlike macrophages, the number and location of T-lymphocytes did not correlate with hemosiderin, suggesting an independent cell-mediated immunological mechanism in bAVM pathogenesis.

Previously, immune cells were mostly analyzed in ruptured $^{[24]}$ and irradiated ${ }^{[25]}$ bAVMs. Our previous study showed that adaptive immune cells were rarely observed in unruptured bAVM. ${ }^{[13]}$ We found in this study that many T-lymphocytes were present in unruptured, previously untreated bAVMs. The possible reason for the discrepancy is that we used a different immunohistochemical staining procedure in this study. Previously, we incubated sections in $0.3 \%$ $\mathrm{H}_{2} \mathrm{O}_{2}$ in methanol to quench the activity of endogenous peroxidase. However, lymphocyte surface markers have been shown to be sensitive to methanol/ $\mathrm{H}_{2} \mathrm{O}_{2}$ treatment. Treating sections with $0.3 \% \mathrm{H}_{2} \mathrm{O}_{2}$ in methanol can reduce our ability to detect membrane markers on frozen sections, ${ }^{[26]}$ and thus, we used $0.3 \% \mathrm{H}_{2} \mathrm{O}_{2}$ in PBS in this study. The case selection could also be responsible for the discrepancy.

Humoral immunity has been reported to play an important role in cerebral cavernous malformation, which might be due to chronic deposition of iron and blood degradation products. ${ }^{[22,27,28]}$ Consistent with this view, we found that plasma cells were present mainly in specimens that had hemosiderin deposition. However, we cannot draw any conclusion regarding adaptive immune responses to the presence of iron from our small descriptive study.

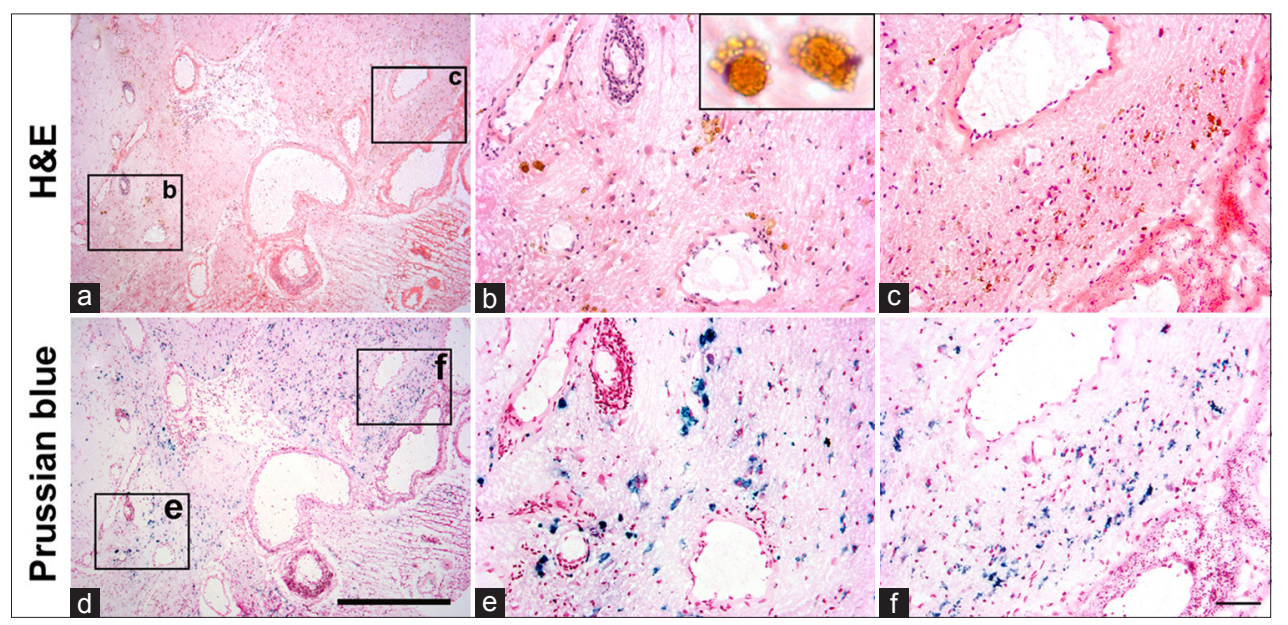

Figure 1: Hemosiderin deposition in unruptured brain arteriovenous malformations. $\mathrm{H}$ and $\mathrm{E}(\mathrm{a}-\mathrm{c})$ and prussian blue staining (d-f) on the adjacent sections. (b) and (c) are enlarged pictures of the regions in squares (b) and (c) in (a) showing hemosiderin-positive areas. Insert in (b) shows two hemosiderin-laden macrophages. (d) Prussian blue staining of an adjacent section of (a). (e) and (f) are enlarged images of the regions in squares (e) and (f) in (d). Scale bars for a and d: $500 \mu \mathrm{m}$; for $b, c, e$ and $f: 50 \mu m$ 


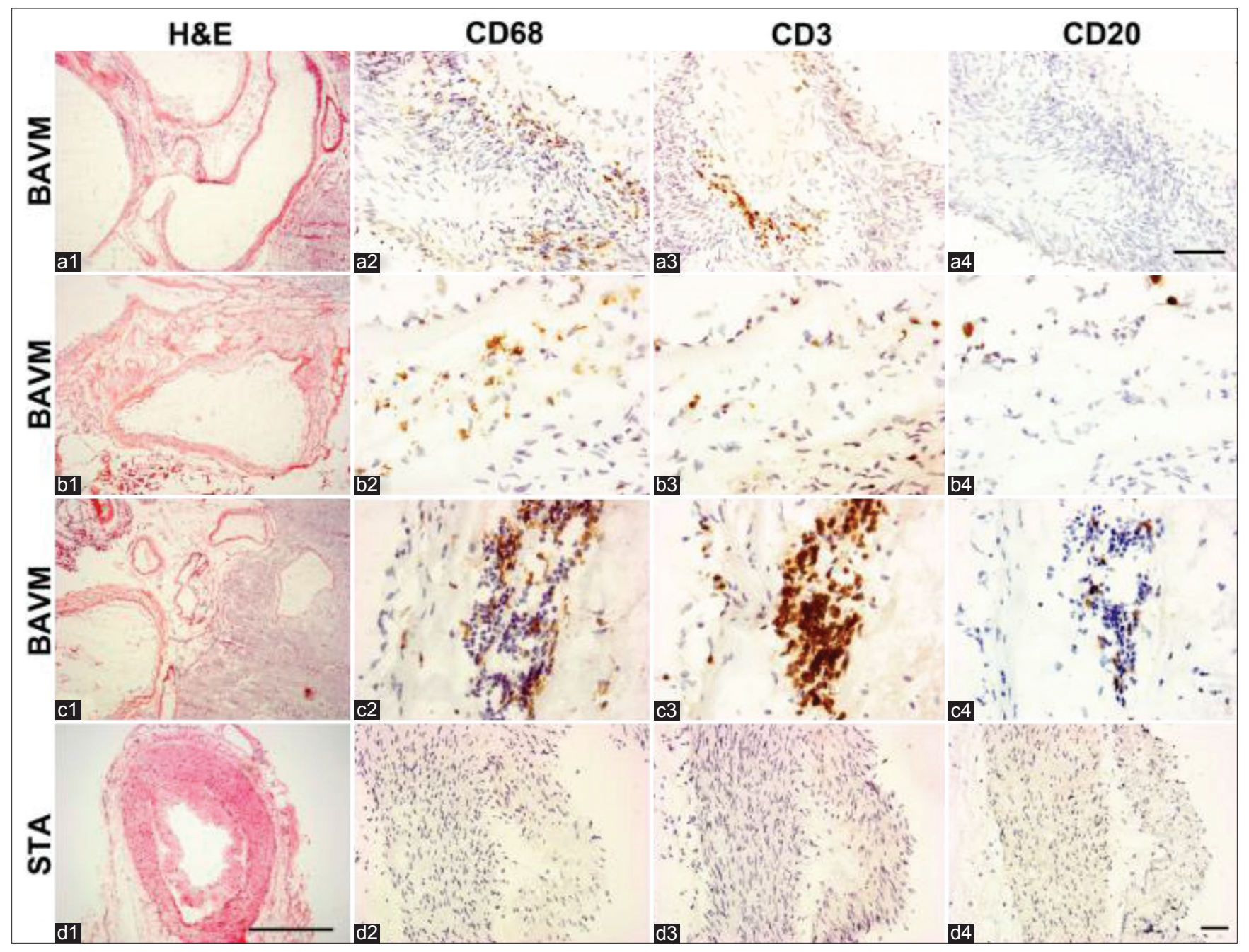

Figure 2: $\mathrm{CD}^{+}$T-lymphocytes and CD68+ macrophages. (a-c) Sections from 3 individual bAVM specimens. (c) Sections from an superficial temporal arteries (STA). Squares in $\mathrm{H}$ and E, images are enlarged to show CD68, CD3 and CD20 positive cells in the images next to them. T-lymphocytes and macrophages were detected on the vessel wall (a2 and a3) and between vessels (b2, c2, b3 and c3). Only a few CD20+ B-lymphocytes were detected in the lumen (b4) and between vessels (c4). No T- and B-lymphocyte, and macrophage were detected on the wall of STA. Scale bar for a1-d1: $500 \mu \mathrm{m}$; scale bar for a2-a4: $100 \mu \mathrm{m}$; scale bar for b2-b4, c2-c4, d2-d4: $20 \mu \mathrm{m}$

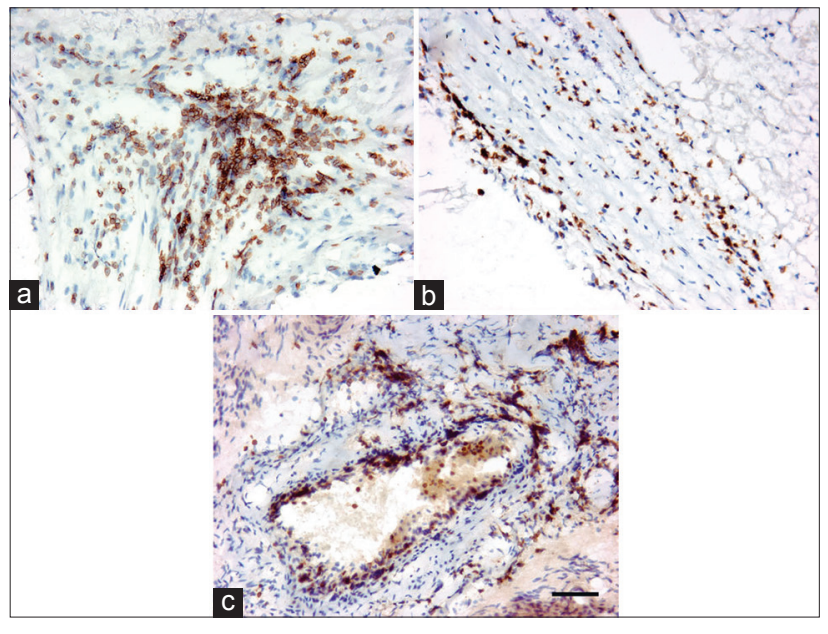

Figure 3: Location of $\mathrm{CD}^{+}$T-lymphocytes. T-lymphocytes were distributed in the perivascular region (a), in the vessel wall (b), and on the surface of the endothelial lining (c). Scale bar: $50 \mu \mathrm{m}$

Our study was underpowered to detect a difference in macrophage loads between hemosiderin-positive and negative specimens, although our data show a strong trend toward that hemosiderin-positive specimens

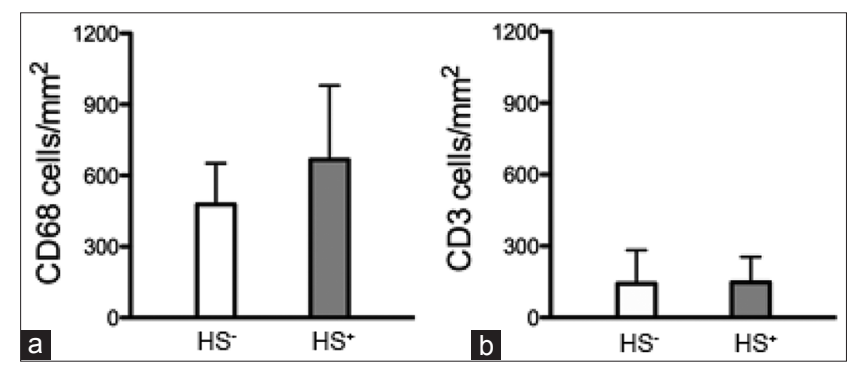

Figure 4: Quantification of inflammatory cells in brain arteriovenous malformation (bAVM). (a) Bar graph shows a trend towards more $\mathrm{CD}^{+} 8^{+}$cells in hemosiderinpositive (HS+) bAVMs than in hemosiderin-negative samples (HS-). (b) Bar graph shows that the numbers of $\mathrm{CD}^{+} \mathrm{T}$-cells were similar in hemosiderin-positive (HS+) and negative (HS-) samples

having more macrophages $(P=0.11)$. The most important finding, however, was that macrophages were present even in the hemosiderin-negative specimens, suggesting that the presence of macrophages is not merely a response to hemorrhage and iron deposition. What remains to be determined is whether the baseline level of macrophage load is causally related to the formation of micro-hemorrhage (e.g. will bAVM with 
high macrophage burden develop micro-hemorrhage?). This will be difficult to test in human studies, and would probably be best addressed in an animal model. An animal study has shown that in bAVM, vessel integrity is impaired. ${ }^{[11]}$ Therefore, the macrophages in bAVM could also be a response to the extravasation of blood content.

One limitation of the study is that we only used one marker for each cell-type. Adding additional markers, including positive and negative controls, would make our data more convincing. However, the markers we used in this study are the most commonly used for macrophages, total lymphocytes, T- and B-lymphocytes, and plasma cells. A future study will employ more markers to confirm the cell-types we have identified here, and to define the subtypes of T- or B-lymphocytes or other inflammatory cells.

In summary, we found that the load and location of T-lymphocytes were not associated with hemosiderin and macrophages. Macrophages are present in unruptured and previously untreated bAVMs, and their load was greater when hemosiderin is present. However, the presence of macrophages is not uniquely driven by hemosiderin, because they were also found in hemosiderin-negative specimens. Future studies need to be conducted to determine (1) how macrophages and lymphocytes contribute to the pathogenesis and progression of the disease, and (2) whether the burden of these cell loads is causally related to the development of micro-hemorrhage, and ultimately, clinically symptomatic hemorrhage.

\section{ACKNOWLEDGMENTS}

The authors would like to thank Voltaire Gungab for assistance with manuscript preparation, and members of the UCSF bAVM Study Project (http://avm.ucsf.edu.) for their support.

\section{REFERENCES}

1. Ruíz-Sandoval JL, Cantú C, Barinagarrementeria F. Intracerebral hemorrhage in young people: analysis of risk factors, location, causes, and prognosis. Stroke 1999;30:537-41.

2. Choi JH, Mohr JP. Brain arteriovenous malformations in adults. Lancet Neurol 2005;4:299-308.

3. Fullerton HJ, Achrol AS, Johnston SC, McCulloch CE, Higashida RT, Lawton MT, Sidney S, Young WL. Long-term hemorrhage risk in children versus adults with brain arteriovenous malformations. Stroke 2005;36:2099-104.

4. Davidson AS, Morgan MK. The embryologic basis for the anatomy of the cerebral vasculature related to arteriovenous malformations. J Clin Neurosci 2011;18:464-9.

5. Kim H, Su H, Weinsheimer S, Pawlikowska L, Young WL. Brain arteriovenous malformation pathogenesis: a response-to-injury paradigm. Acta Neurochir Suppl 2011;111:83-92.

6. Kim H, Pawlikowska L, Young WL. Genetics and vascular biology of brain vascular malformations. In: Mohr JP, Wolf PA, Grotta JC, Moskowitz MA, Mayberg MR, von Kummer R, editors. Stroke: pathophysiology, Diagnosis, and Management. $5^{\text {th }}$ ed., Ch. 12 Philadelphia: Churchill Livingstone Elsevier; 2011. p. 169-86.

7. Kim H, Marchuk DA, Pawlikowska L, Chen Y, Su H, Yang GY, Young WL. Genetic considerations relevant to intracranial hemorrhage and brain arteriovenous malformations. Acta Neurochir Suppl 2008;105:199-206.

8. Choi EJ, Chen W, Jun K, Arthur HM, Young WL, Su H. Novel brain arteriovenous malformation mouse models for type 1 hereditary hemorrhagic telangiectasia. PLoS One 2014;9:e88511.

9. Moftakhar P, Hauptman JS, Malkasian D, Martin NA. Cerebral arteriovenous malformations. Part 1: cellular and molecular biology. Neurosurg Focus 2009;26:E10.

10. Chen W, Guo Y, Walker EJ, Shen F, Jun K, Oh SP, Degos V, Lawton MT, Tihan T, Davalos D, Akassoglou K, Nelson J, Pile-Spellman J, Su H, Young WL. Reduced mural cell coverage and impaired vessel integrity after angiogenic stimulation in the Alk1-deficient brain. Arterioscler Thromb Vasc Biol 2013;33:305-10.

11. Chen W, Sun Z, Han Z, Jun K, Camus M, Wankhede M, Mao L, Arnold T, Young WL, Su H. De novo cerebrovascular malformation in the adult mouse after endothelial Alk1 deletion and angiogenic stimulation. Stroke 2014;45:900-2.

12. Chen W, Choi EJ, McDougall CM, Su H. Brain arteriovenous malformation modeling, pathogenesis, and novel therapeutic targets. Transl Stroke Res 2014;5:316-29.

13. Chen Y, Zhu W, Bollen AW, Lawton MT, Barbaro NM, Dowd CF Hashimoto T, Yang GY, Young WL. Evidence of inflammatory cell involvement in brain arteriovenous malformations. Neurosurgery 2008;62:1340-9.

14. Chen Y, Fan Y, Poon KY, Achrol AS, Lawton MT, Zhu Y, McCulloch CE, Hashimoto T, Lee C, Barbaro NM, Bollen AW, Yang GY, Young WL. MMP-9 expression is associated with leukocytic but not endothelial markers in brain arteriovenous malformations. Front Biosci 2006;11:3121-8.

15. Chen Y, Pawlikowska L, Yao JS, Shen F, Zhai W, Achrol AS, Lawton MT, Kwok PY, Yang GY, Young WL. Interleukin-6 involvement in brain arteriovenous malformations. Ann Neurol 2006:59:72-80

16. Hashimoto G, Inoki I, Fujii Y, Aoki T, Ikeda E, Okada Y. Matrix metalloproteinases cleave connective tissue growth factor and reactivate angiogenic activity of vascular endothelial growth factor 165. J Biol Chem 2002;277:36288-95.

17. Storer KP, Tu J, Karunanayaka A, Morgan MK, Stoodley MA. Inflammatory molecule expression in cerebral arteriovenous malformations. J Clin Neurosci 2008;15:179-84.

18. Hasan DM, Amans M, Tihan T, Hess C, Guo Y, Cha S, Su H Martin AJ, Lawton MT, Neuwelt EA, Saloner DA, Young WL. Ferumoxytol-enhanced MRI to image inflammation within human brain arteriovenous malformations: a pilot investigation. Transl Stroke Res 2012:3:166-73.

19. Prayer L, Wimberger D, Stiglbauer R, Kramer J, Richling B, Bavinzski G, Czech T, Imhof H. Haemorrhage in intracerebral arteriovenous malformations: detection with MRI and comparison with clinical history. Neuroradiology 1993;35:424-7.

20. Yousem DM, Flamm ES, Grossman RI. Comparison of MR imaging with clinical history in the identification of hemorrhage in patients with cerebral arteriovenous malformations. AJNR Am J Neuroradiol 1989;10:1151-4.

21. Guo Y, Saunders T, Su H, Kim H, Akkoc D, Saloner DA, Hetts SW, Hess C, Lawton MT, Bollen AW, Pourmohamad T, McCulloch CE, Tihan T, Young WL. Silent intralesional microhemorrhage as a risk factor for brain arteriovenous malformation rupture. Stroke 2012;43:1240-6.

22. Shi C, Shenkar R, Du H, Duckworth E, Raja H, Batjer HH, Awad IA Immune response in human cerebral cavernous malformations. Stroke 2009;40:1659-65.

23. Halim AX, Singh V, Johnston SC, Higashida RT, Dowd CF, Halbach VV, Lawton MT, Gress DR, McCulloch CE, Young WL. Characteristics of brain arteriovenous malformations with 
coexisting aneurysms: a comparison of two referral centers. Stroke 2002;33:675-9.

24. Aziz MM, Takagi Y, Hashimoto N, Miyamoto S. Activation of nuclear factor kappaB in cerebral arteriovenous malformations. Neurosurgery 2010;67:1669-79.

25. Redekop GJ, Elisevich KV, Gaspar LE, Wiese KP, Drake CG. Conventional radiation therapy of intracranial arteriovenous malformations: long-term results. J Neurosurg 1993;78: 413-22.

26. Adams RA, Schachtrup C, Davalos D, Tsigelny I, Akassoglou K. Fibrinogen signal transduction as a mediator and therapeutic target in inflammation: lessons from multiple sclerosis. Curr Med Chem 2007; 14:2925-36.

27. Shenkar R, Shi C, Check IJ, Lipton HL, Awad IA. Concepts and hypotheses: inflammatory hypothesis in the pathogenesis of cerebral cavernous malformations. Neurosurgery 2007;61:693-702.

28. Shi C, Shenkar R, Batjer HH, Check IJ, Awad IA. Oligoclonal immune response in cerebral cavernous malformations. Laboratory investigation. J Neurosurg 2007;107:1023-6.

Cite this article as: Guo $\mathrm{Y}$, Tihan $\mathrm{T}$, Kim H, Hess C, Lawton MT, Young WL, Zhao YL, Su H. Distinctive distribution of lymphocytes in unruptured and previously untreated brain arteriovenous malformation. Neuroimmunol Neuroinflammation 2014;1(3):147-52.

Source of Support: This study was supported by National Institutes of Health grants: P01 NS44155 (WLY and HS), R21 NS083788 (HS), R01 NS027713 (HS), and R01 NS034949 (HK). Hebei Provincial Natural Science Foundation of China grant: H2013201283 (YG). National Natural Science Foundation of China grant: No. 81271313 (YLZ, YG). Conflict of Interest: No.

Received: 01-08-2014; Accepted: 01-09-2014 